La narration dans l'espace de la coupole : une rencontre compliquée? Autour des mosaïques du baptistère de San Giovanni in Fonte à Naples (fin $\mathrm{IV}^{\mathrm{e}}$-début $\mathrm{V}^{\mathrm{e}}$ siècle)

\title{
Chiara Croci
}

\section{OpenEdition}

\section{Journals}

Édition électronique

URL : http://journals.openedition.org/edl/1139

DOI : $10.4000 /$ edl. 1139

ISSN : 2296-5084

\section{Éditeur}

Université de Lausanne

\section{Édition imprimée}

Date de publication : 15 mai 2018

Pagination : 65-84

ISBN : 978-2-940331-68-0

ISSN : 0014-2026

\section{Référence électronique}

Chiara Croci, «La narration dans l'espace de la coupole : une rencontre compliquée ? Autour des

mosaïques du baptistère de San Giovanni in Fonte à Naples (fin IVe-début Ve siècle) », Études de lettres [En ligne], 2 | 2018, mis en ligne le 15 mai 2020, consulté le 13 décembre 2020. URL : http:// journals.openedition.org/edl/1139; DOI : https://doi.org/10.4000/edl.1139 


\section{LA NARRATION DANS L'ESPACE DE LA COUPOLE: UNE RENCONTRE COMPLIQUÉE? AUTOUR DES MOSAÏQUES DU BAPTISTÈRE DE SAN GIOVANNI IN FONTE À NAPLES (FIN IVe-DÉBUT Ve SIÈCLE)}

Lieu par excellence des images théophaniques, la coupole a réservé, spécialement dans le monde médiéval et byzantin, peu d'espace à la narration. En accueillant des scènes issues du Nouveau Testament disposées dans un schéma radial, la coupole du baptistère de Naples constitue en ce sens une exception qui mérite d'être approfondie. Des considérations sur l'iconographie des mosaïques et leur articulation montrent comment on a su exploiter l'espace afin d'y aménager des paradigmes bibliques adaptés à un contexte spécifique et permettent d'émettre des hypothèses sur le maigre succès de la narration dans les bâtiments à coupole.

Emblème du ciel, la coupole s'avère être, de par son emplacement, sa structure et sa portée symbolique, un espace destiné à la représentation du transcendant (fig. 1). Toutefois, sa forme circulaire et concave la rend moins adaptée à accueillir des cycles narratifs, dont la multitude d'épisodes se succède plus aisément sur des parois planes. Dans le monde médio-byzantin, où l'architecture à coupole a eu un succès plus important que dans l'Occident médiéval, l'on observe facilement cette tendance: les coupoles des églises sont habituellement dominées par le buste du Christ entouré de saints, ou par une scène théophanique - comme l'Ascension ou la Pentecôte - disposée de manière concentrique, alors que les scènes narratives sont reléguées dans la partie inférieure du bâtiment (fig. 2). Après quelques prémices antiques et paléochrétiennes, la présence d'un cycle narratif dans l'espace d'une 
Fig. 1 - Naples, Battistero di San Giovanni in Fonte, fin $\mathrm{IV}^{\mathrm{e}}$-début $\mathrm{V}^{\mathrm{e}}$ siècle, coupole.

coupole n'est effectivement attestée qu'au XIII ${ }^{\mathrm{e}}$ siècle, dans le narthex de Saint-Marc à Venise ${ }^{1}$ (fig. 3). La coupole du baptistère de San Giovanni in Fonte à Naples (fin $\mathrm{IV}^{\mathrm{e}}$-début $\mathrm{V}^{\mathrm{e}}$ siècle), organisée grâce à huit festons de fruits, plantes et oiseaux entourant un médaillon sommital, présente une série d'épisodes issus en grande partie du Nouveau Testament - la Samaritaine, les Noces de Cana, les Femmes au Tombeau, la Pêche Miraculeuse, Pierre sauvé des eaux et l'ainsi dite Traditio legis - et constitue en ce sens un lieu d'observation privilégié pour étudier la question des images narratives dans les édifices en plan centré (fig. 1).

I. A. Grabar, «La décoration des coupoles à Karye Camii et les peintures italiennes du Dugento». 
Fig. 2 - Daphni, Église de la Dormition, coupole, vers 1100.

\section{Le cas de Naples parmi les systèmes décoratifs des coupoles}

Les développements de la matière narrative dans les coupoles découlent principalement de l'existence de deux systèmes d'organisation de la décoration. Celle-ci peut s'articuler par exemple dans un large bandeau autour du médaillon central: une solution aux racines anciennes attestée par la présence du banquet funéraire en frise continue qui entoure le médaillon central du tholos thrace de Kazanlak (fin IV e-début $\mathrm{III}^{\mathrm{e}}$ s. av. J.-C.) et qui sera reprise pour agencer des scènes de l'Ancien et du Nouveau Testament dans la coupole du mausolée de la Paix de 
Fig. 3 - Venise, Saint-Marc, atrium, coupole de Moïse, XIII ${ }^{\mathrm{e}}$ siècle.

El-Bagawat ( $1^{\text {re }}$ moitié $\mathrm{V}^{\mathrm{e}}$ siècle $)^{2}$. Ce système, qui s'adapte à l'illustration d'une histoire continue, sera notamment exploité pour représenter le cycle de la vie de Moïse dans une des coupoles du narthex marcien ${ }^{3}$ (fig. 3). L'espace de la coupole peut aussi être divisé en une série de

2. G. Cipriano, El-Bagawat, p. 195-236, tav. 28-31.

3. Pour la datation, l'iconographie et la composition de ces mosaïques, cf. O. Demus, The mosaics of San Marco in Venice, II, p. 130-140, 169-177, tav. 73. 
secteurs triangulaires agencés autour d'un médaillon sommital ${ }^{4}$. Attesté dans la voûte de la Domus delle Volte Dipinte d'Ostie (II ${ }^{\mathrm{e}}$ siècle), ce schéma se prête à une disposition paratactique des éléments: on s'en servira donc pour y juxtaposer des figures iconiques. Expérimentée dans la coupole de Sant'Aquilino à Milan (fin IVe-début Ve siècle), cette solution sera amplement développée par la suite, comme le montrent les églises de Pammakaristos et de Saint-Sauveur-in-Chora à Constantinople $\left(\mathrm{XIV}^{\mathrm{e}} \text { siècle }\right)^{5}$. Moins adapté à la narration d'une histoire continue, l'agencement radial n'est pourtant pas complètement inconnu dans le cas de la représentation de scènes bibliques: le baptistère de Naples trouvait notamment un précédent dans la coupole perdue du mausolée de Santa Costanza à Rome (moitié IV $\mathrm{IV}^{\mathrm{e}}$ siècle) ${ }^{6}$. Dans les deux cas, ce système convenant à la représentation d'une série d'instantanés a été exploité pour réunir des épisodes ayant une symbolique commune, qui ne devaient pas constituer un cycle narratif.

Des considérations sur l'iconographie, la structure et l'articulation du décor de Naples permettent de montrer comment on a su profiter de l'espace de la coupole pour agencer des paradigmes bibliques dans ce contexte spécifique et également de s'interroger sur les raisons du maigre succès de la narration dans ce type de bâtiments. Il convient tout d'abord de relever que la solution de la coupole de San Giovanni in Fonte s'éloigne de ses précédents les plus proches, car elle réunit plus d'un épisode dans le même secteur. Selon les sources qui nous ont transmis le décor perdu de la coupole de Santa Costanza, celle-ci présentait vingt-quatre rayons issus de douze candélabres verticaux, partagés aux trois quarts par des rinceaux, occupés chacun par une scène de l'Ancien Testament dans la partie inférieure et une scène du Nouveau Testament dans la partie supérieure. La même chose est attestée à Centcelles, monument qui se situe au tournant entre les deux principaux systèmes d'organisation relevés, sa coupole étant partagée en trois zones

4. En ce qui concerne les systèmes d'articulation des coupoles d'époque tardoantique, cf. J. J. Rasch, A. Arbeiter, Das Mausoleum der Constantina in Rom, p. 239-280 et la contribution de M. Braconi dans ce volume.

5. J. Lowden, Early Christian and Byzantine art, fig. 247 et 262.

6. Pour le baptistère de Naples, cf. G. Ferri, I mosaici del battistero di San Giovanni in Fonte a Napoli; J.-L. Maier, Le baptistère de Naples et ses mosä̈ques; Ch. Croci, Una "questione campana", p. 48-117. Pour la coupole du mausolée de S. Costanza, cf. en dernier J. J. Rasch, A. Arbeiter, Das Mausoleum der Constantina in Rom. 
horizontales concentriques, et plus précisément en une frise cynégétique continue surmontée par deux registres divisés à leur tour verticalement ${ }^{7}$. Le registre intermédiaire présente des scènes issues des deux Testaments, unies comme à Naples et à Santa Costanza en raison de leur symbolique commune, sans but d'en faire un récit. Celles-ci sont disposées dans des cadres dont la largeur variable s'adapte à la tradition iconographique de l'épisode respectif: les trois Hébreux dans la fournaise occupent donc un secteur plus vaste que Daniel dans la fosse des lions ou le Bon Berger, pour se limiter à un exemple.

La coupole de Naples, par contre, suivant de près la structure octogonale du tambour, présente un schéma "solaire» qui détermine dans une large mesure la disposition des scènes testamentaires. Pour qu'elles soient aménagées, une coulisse est créée dans chaque secteur trapézoïdal grâce aux rideaux écartés sur les côtés descendant des entablements. Les scènes qui restent trahissent un ordre chronologique entre les premiers miracles du Christ (Noces Cana, Samaritaine) et des scènes post mortem ou théophaniques (Pêche miraculeuse, Traditio legis); toutefois, leur critère de sélection demeure, comme pour Santa Costanza et Centcelles, difficile à déterminer. Il s'agit d'une série de paradigmes bibliques unis en raison de leur valeur sotériologique analogue, selon une combinaison paratactique qui reflète la logique de l'initiation baptismale, fondée sur un ensemble de figures paradigmatiques et non pas sur une narration continue. Représentées selon le sermo brevis caractéristique du premier art chrétien, ces scènes instantanées évoquaient un réseau de concepts essentiels de l'initiation baptismale ${ }^{8}$. Le choix de réunir deux scènes distinctes dans le même secteur, au sein d'un programme qui n'imposait pas un nombre précis d'épisodes, est digne de grande attention et

7. A. Arbeiter, D. Korol (Hrsg.), Der Kuppelbau von Centcelles. Cf. fig. 5, p. 27 de la contribution de M. Braconi dans ce volume.

8. Sur la liturgie baptismale paléochrétienne, cf. G. H. Baudry, Le baptême et ses symboles. Un langage très raccourci caractérise les Miracula Christi, tituli historiarum dont la fonction et le rapport aux cycles narratifs peints demeure discutée. L'hypothèse d'y voir les didascalies du programme figuratif d'un baptistère a été mise en discussion par les recherches plus récentes (F. Lubian, «Un caso di riscrittura metrica "ultrabreve” dei Vangeli»), mais le rapport entre ces compositions et le sermo brevis de l'art funéraire et baptismal mériterait d'être approfondi. Sur le sermo brevis dans l'art paléochrétien cf. E. Kitzinger, "Christian imagery", p. 142-147, duquel l'on reprend également le concept de «signitive art» employé par la suite. 
mérite d'être étudié à la lumière de la fonction de l'édifice, ainsi que des contraintes et des potentialités de l'espace de la coupole. Le schéma radial a en effet été exploité de manière singulière afin de mettre en scène le discours fondé sur ce réseau d'instantanés visant à souligner la fonction de l'espace et la portée du rituel qui s’y déroulait.

\section{Solutions narratives «baptismales»}

Dans le secteur qui subsiste du côté nord-est (fig. 4), la Samaritaine, pivot de la composition, tourne le dos aux deux serviteurs des Noces de Cana, représentés dans la partie droite du cadre en train de verser de l'eau dans des vases. Il s'agit de deux épisodes largement attestés dans la peinture et la sculpture funéraire paléochrétienne qui, interprétés sous un prisme baptismal par les Pères de l'Église, devaient faire partie de la liturgie quadragésimale napolitaine ${ }^{9}$. La manière dont ils sont représentés dans ce cas demeure toutefois unique et se détache de leur tradition iconographique respective. En dépit de l'état fragmentaire de la figure du Christ dans la partie gauche du secteur, l'on constate l'absence des éléments qui caractérisent habituellement le dialogue qui a lieu entre celui-ci et la Samaritaine, à savoir la torsion des deux têtes l'une vers l'autre et le geste de parole du Seigneur, attestés par exemple dans le cubiculum $\mathrm{F}$ de la catacombe de la via Latina et dans les mosaïques de la nef de Sant'Apollinare Nuovo à Ravenne ${ }^{10}$. À Naples, par ailleurs, la Samaritaine est complètement frontale et son regard se pose sur le spectateur. Contrairement à l'attitude usuelle, qui la montre en train de puiser l'eau, son action ici est terminée et elle exhibe le récipient déjà rempli. En même temps, le geste du Christ ne semble pas être adressé à la femme, mais à la cruche d'eau: il indique en effet le récipient comme s'il cherchait à le soutenir.

L'épisode voisin trahit une formule iconographique encore plus singulière: les deux serviteurs ne sont pas accompagnés par le Christ, véritable moteur du miracle de Cana, représenté généralement seul ou flanqué de serviteurs en train d'accomplir la transformation de l'eau en vin. On a proposé d'expliquer cette solution iconographique par le manque

9. Voir à ce propos K. Gandolfi, «Les mosaïques du baptistère de Naples», p. 25.

Io. Cf. Ch. Croci, Una "questione campana", fig. 84-85. 
Fig. 4 - Naples, Battistero di San Giovanni in Fonte, fin IVe-début $V^{\mathrm{e}}$ siècle, coupole, La Samaritaine et Les Noces de Cana. 
Fig. 5 - Naples, Battistero di San Giovanni in Fonte, fin IV e-début $V^{e}$ siècle, coupole, La pêche miraculeuse et Pierre sauvé des eaux. 
d'espace, qui aurait mené à ne représenter le Christ qu'une seule fois pour les deux épisodes ${ }^{11}$. Toutefois, en dépit de l'état fragmentaire de la figure, le Christ, de par sa position et son attitude, participe clairement à l'épisode qui se déroule au puits de Sichem. La solution iconographique choisie pour la partie droite du secteur s'avère donc être le fruit d'un choix précis, visant à mettre l'accent sur l'élément qui incarne le caractère extraordinaire de l'épisode et sa portée baptismale, à savoir l'eau. Par la transposition de deux épisodes répandus dans les arts mineurs à l'échelle monumentale, leur caractère synthétique, abrégé, en a même été accentué. L'environnement et l'action des personnages sont réduits au minimum, afin d'insister sur le pouvoir de l'eau: un élément vivant, en mesure de transformer et régénérer. Il n'est d'ailleurs pas étonnant que l'on ait fait allusion à la transformation de l'eau en vin à Cana dans la prière de consécration de l'eau de la piscine baptismale ${ }^{12}$.

Le choix d'échapper au schéma radial en représentant deux épisodes distincts dans le même cadre peut donc s'expliquer à la lumière d'un processus visant à adapter la décoration au domaine baptismal. Si la division de la coupole en huit espaces trapézoïdaux était, à première vue, contraignante pour l'agencement du programme, on a su ici l'exploiter afin d'unir des instantanés complémentaires, en facilitant leur identification et en stimulant les processus d'association et de comparaison visés par cette narration syncopée caractéristique du premier art chrétien. Pour représenter deux épisodes dans un même secteur, sans division, l'on ne disposait vraisemblablement pas de modèles établis à l'échelle monumentale. La peinture des catacombes n'offrait pas non plus de références vu qu'un cadre unique était habituellement réservé à ce type d'épisodes ${ }^{13}$. Les deux scènes de la coupole napolitaine sont donc unies sans solution de continuité et se distinguent grâce au fait que la Samaritaine et le serviteur voisin sont dos à dos: un procédé amplement attesté pour représenter les mêmes paradigmes salvifiques sur les sarcophages à frise continue (fig. 6). Par ailleurs, la séparation des deux épisodes est soulignée grâce aux tesselles plus sombres des habits et de la peau qui éloignent les serviteurs en deuxième plan: cette logique de placer les figures dans l'espace

II. G. Ferri, I mosaici del battistero di San Giovanni in Fonte a Napoli, p. 70.

I2. J.-L. Maier, Le baptistère de Naples et ses mosaïques, p. 89.

13. Cf. par exemple la voûte du cubiculum 69 de la Catacombe de Domitilla. N. Zimmermann, Werkstattgruppen römischer Katakombenmalerei, p. 135-139. 


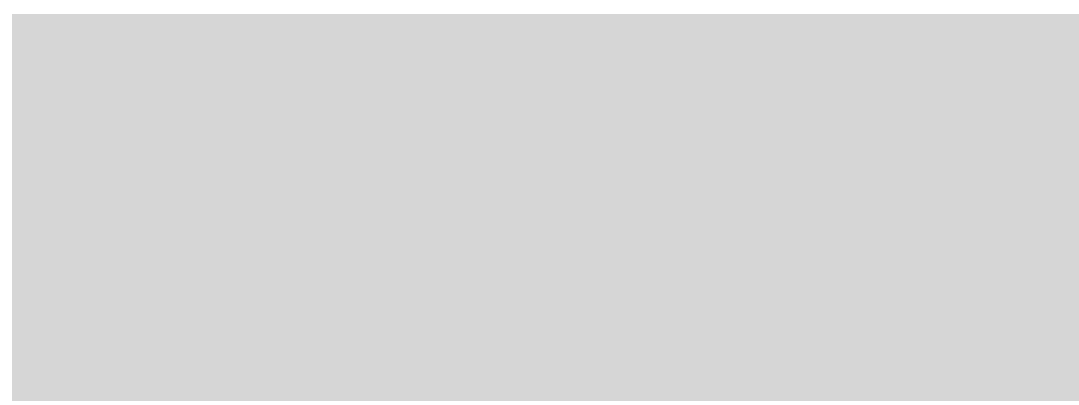

Fig. 6 - Rome, Museo Pio Cristiano, Sarcophage, deuxième quart IVe siècle.

est attestée dans les bas-reliefs antiques et sera notamment développée sur les sarcophages du IV ${ }^{\mathrm{e}}$ siècle $^{14}$.

Dans le secteur fragmentaire où sont superposés l'épisode de Pierre sauvé des eaux (Mt XIV) et la Pêche miraculeuse (Jn XXI, 3-7; fig. 5), l'exploitation du schéma radial de la coupole est même plus significative $^{15}$. Les deux scènes ont encore une fois une portée baptismale claire: elles indiquent la nécessité de s'en remettre au Christ pour être sauvé et se déroulent dans une ambiance lacustre. Ces épisodes étaient donc présentés comme des métaphores du baptême par les Pères de l'Église et faisaient partie des lectures quadragésimales ${ }^{16}$. Par rapport à la Samaritaine et aux Noces de Cana, l'iconographie de ces deux miracles ne se prêtait pas à être raccourcie ultérieurement, leur mise en scène ne pouvant pas se passer du paysage lacustre et du Christ, accompagné par Pierre dans un cas, et par la barque des pêcheurs dans l'autre. Toutefois, leur superposition dans un même secteur s'explique facilement: en restant fidèle à la tradition iconographique d'épisodes impliquant un déroulement longitudinal, l'espace du cadre n'aurait pas pu être exploité convenablement par une seule scène. L'union des deux miracles sur le lac de Tibériade, motivée sans doute par l'ambiance analogue, entraîne une interruption de l'apparente disposition chronologique des scènes du programme restant: la scène de Pierre sauvé des Eaux, une des dernières étapes de la

I4. Cf. par exemple Rep. III, 38 et 65.

I5. J. Wilpert (Die römischen Mosaiken und Malereien der kirchlichen Bauten vom IV. bis XIII. Jahrhundert, fig. 68) propose une reconstruction hypothétique qui peut être acceptée dans ses traits essentiels.

I6. Cf. par exemple K. Gandolfi, «Les mosaïques du baptistère de Naples», p. 25 sq. 
prédication en Galilée, a en effet été associée pour des raisons de lisibilité à la Pêche Miraculeuse, vraisemblablement celle survenue quand le Christ était déjà ressuscité. La disposition de ces épisodes, superposés apparemment en chiasme, favorisait certainement leur identification et leur interprétation. Elle participait donc à la formation du réseau sémantique visé avec la représentation d'une série de paradigmes bibliques de ce type. Cette disposition spéculaire de deux épisodes ne trouve en réalité pas de parallèles dans la sculpture ni dans la peinture funéraire de la même époque, mais elle reviendra dans des cycles narratifs monumentaux, comme celui de Santa Maria Maggiore à Rome (432-440) ${ }^{17}$ (fig. 7). Les deux miracles lacustres, difficiles à synthétiser, ont donc été unis selon une logique pertinente à la succession d'épisodes narratifs dans un cycle monumental: une circonstance qui pourrait peut-être s'expliquer par la référence commune constituée par la production de livres enluminés ${ }^{18}$. Si à Rome, comme dans certaines pages de la Genèse de Vienne, la composition en chiasme entraîne un regard circulaire qui embrasse le déroulement de la narration (de droite à gauche en haut et de gauche à droite en bas), les scènes de Naples demeurent par contre deux instantanés autonomes qui ne présupposaient pas un sens de lecture précis ${ }^{19}$.

L'allure - au premier abord - narrative des deux secteurs analysés est par contre dépassée dans le cadre suivant, traité comme une petite abside pour y représenter, grâce à une composition triangulaire parfaitement adaptée à l'espace disponible, l'ainsi dite Traditio legis. La représentation de cette scène, née selon toute vraisemblance pour orner un précédent absidal, rentre dans le processus de sélection des paradigmes les plus significatifs dans le contexte baptismal qui est à la base de ce programme. Image de la majesté et de l'efficacité salvifique du Christ et de sa nouvelle loi, la Traditio legis s'affirme comme le manifeste par excellence de l'art signitif des premiers siècles: il n'est pas surprenant qu'elle dominait la

I7. Sur ce cycle, cf. M. R. Menna, «I mosaici di Santa Maria Maggiore», fig. 8.

I8. Un lien plus général entre les mosaïques de Naples et les enluminures est suggéré par G. Ferri, I mosaici del battistero di San Giovanni in Fonte a Napoli, p. 82. Sur le rapport entre art monumental et enluminure, ainsi que sur la question des «guides iconographiques", cf. E. Kitzinger, "The role of miniature painting in mural decoration", p. $115-121$.

I9. Voir par exemple le folio 13r de la Genèse de Vienne (Wien, Österreichische Nationalbibliothek, cod. theol. gr. 31). 
Fig. 7 - Rome, Santa Maria Maggiore (432-440), mosaïques de la nef, Rachel et Jacob; Jacob et Laban.

série de paradigmes salvifiques se déployant sur les sarcophages en frise continue de la seconde moitié du IV ${ }^{\mathrm{e}}$ siècle. Privilégiant notamment un caractère instantané, en mesure de favoriser la lecture simultanée et comparative des épisodes, on a su, dans ce programme, gérer au mieux l'espace et trouver pour chaque scène la solution «narrative» la plus efficace: cela a amené à des résultats parfois plus proches de la sculpture funéraire $\mathrm{du} \mathrm{IV}^{\mathrm{e}}$ siècle et parfois à un langage pertinent à l'art monumental, narratif ou iconique. Cas exemplaire où le schéma «solaire» de la coupole a été exploité pour construire un discours sur la fonction de l'espace et la portée du baptême, San Giovanni in Fonte s'avère être un unicum, 
dont la veine expérimentale peut s'expliquer à la lumière du processus de transposition dans un tel contexte des paradigmes enracinés dans les arts mineurs, peu diffusés à l'échelle monumentale.

\section{Coupoles, baptistères, scènes narratives: aux sources d'un insuccès}

La solution de Naples peine à trouver des échos successifs, notamment dans le domaine baptismal: des cycles narratifs vont retourner dans la coupole des baptistères médiévaux de Parme et de Padoue, mais il s'agit de bâtiments qui, de par leurs dimensions et leur spectre fonctionnel, appartiennent désormais à un tout autre horizon ${ }^{20}$. L'échantillon restreint de témoignages conservés permet cependant de constater que, après le cas particulier de Doura Europos (milieu du III ${ }^{\mathrm{e}}$ siècle), les baptistères contemporains, ou à peine postérieurs à celui de Naples, ont privilégié des programmes théophaniques pour la décoration de leurs coupoles. Dans le baptistère des Orthodoxes de Ravenne (milieu du $\mathrm{V}^{\mathrm{e}}$ siècle), les paradigmes salvifiques sont destinés à la partie inférieure de l'édifice, alors que le baptême du Christ, situé dans le clipeus sommital, apparaît comme une théophanie plongée dans une dimension céleste occupée par les apôtres offrant des couronnes, des trônes vides et des autels ${ }^{21}$. Le baptistère de la cathédrale de Milan devait également présenter un décor théophanique, centré sur le staurogramme, qui trouvait peut-être son écho dans la mosaïque du baptistère d'Albenga ${ }^{22}$.

L'insuccès des instantanés salvifiques que l'on remarque, de manière générale, dans la décoration des édifices à coupole, trouve des raisons liées à la structure et aux conditions de visibilité. Cela est d'autant plus évident dans le cas des baptistères: les personnes qui rentraient dans un édifice comme celui de Naples, pour se soumettre à un rite qui, encore au $\mathrm{V}^{\mathrm{e}}$ siècle, se déroulait la nuit, auraient dans un premier temps distingué uniquement le schéma radial créé par les festons entourant le

20. Voir par exemple L. Geymonat, "Parma 1233».

2I. Sur les mosaïques du baptistère des orthodoxes: F. W. Deichmann, Ravenna, vol. I, p. 138-151; vol. II.1, p. 31-46; vol. III, p. 36-39.

22. Voir à ce propos la contribution de A. Filipová dans ce volume. Cf. aussi Paulin de Nole, Carmina, XXVIII, 180-184, qui parle d'une ambiance du complexe de Cimitile, ayant selon toute vraisemblance fonction baptismale et étant ornée par un stellato tholo. 
staurogramme sommital. En dépit de leur caractère immédiat, les scènes dans les huit secteurs demandaient une contemplation plus longue pour saisir leur concept implicite, mais incompatible avec le regard des participants à un rituel frénétique, destiné par ailleurs à être abrégé au fil des siècles. Un espace comme le cathecumenum, où les candidats demeuraient plus longuement et où ils auraient pu s'attarder sur des images reflétant les concepts fondateurs de l'initiation qu'ils étaient appelés à répéter avant de se soumettre au rituel, se serait mieux prêté à la représentation d'un scénario de ce type. Jonas, l'Orante et le Bon Pasteur habitaient notamment le pavement de la basilique théodorienne méridionale du complexe d'Aquilée (313-319), qui avait peut-être une fonction catéchuménale. Une série de figures paradigmatiques, associées pour leur valeur sotériologique commune et non dans le but de raconter une histoire, se retrouve notamment dans le programme iconographique des portes de Santa Sabina à Rome (432-440), qui s'ouvraient selon toute vraisemblance sur un atrium destiné à l'initiation des catéchumènes ${ }^{23}$.

Si l'apparent insuccès de la narration dans les édifices à coupole, même dans la forme du sermo brevis, trouve des raisons dans la fonction des bâtiments, ainsi que dans leurs particularités structurelles, il est indéniable qu'il se doit également aux enjeux profonds du tournant qui a investi la production artistique entre la fin du IV et le début du V $\mathrm{V}^{\mathrm{e}}$ siècle, lorsque les images se sont imposées dans les édifices de culte. Face au défi de concevoir des projets figuratifs à l'échelle monumentale, on a souvent préféré des solutions plus solennelles et iconiques, visant à mettre en scène une autre image du Sauveur: le thaumaturge de la peinture et de la sculpture funéraire n'était désormais plus suffisant ${ }^{24}$. Alors que dans les édifices de culte des véritables cycles narratifs bibliques iront côtoyer les programmes théophaniques, souvent inspirés par la vision apocalyptique, les images salvifiques demeureront du ressort des arts mineurs. C'est ainsi que pour la coupole d'un baptistère, les paradigmes bibliques

23. Sur le pavement d'Aquilée, cf. G. Pelizzari, Il pastore ad Aquileia; sur les portes de Sainte Sabine et la fonction de son atrium, cf. I. Foletti, M. Gianandrea, Zona liminare. Le parallèle avec Sainte Sabine (ainsi que les précédents de Santa Costanza et Centcelles) oblige à s'interroger sur l'apparente absence de scènes de l'Ancien Testament de la coupole de Naples. Partie intégrante de la catéchèse baptismale, il n'est pas exclu que des images vétérotestamentaires trouvaient place notamment dans des espaces annexes au baptistère, voire dans la basilique même.

24. Sur ces développements, cf. plus en général J.-M. Spieser, Images du Christ. 
étant relégués dans la partie inférieure ou dans des pièces annexes, on concevra des images en mesure de montrer un Christ présent au moment du rite, dans lequel le candidat aurait pu se refléter. Fondé sur le langage signitif du premier art chrétien et tourné en même temps vers l'imagerie visionnaire qui dominera l'art monumental des siècles successifs, le baptistère de Naples s'impose comme une étape charnière de la civilisation artistique de l'Antiquité tardive et du haut Moyen Âge.

Chiara Croci

Université de Lausanne, Section d'histoire de l'art 


\section{BIBLIOGRAPHIE}

\section{Source}

Paulin de Nole, Carmina - Paolino di Nola, I Carmi, a cura di Andrea Ruggiero, Roma, Città Nuova Editrice, 1990.

\section{Études}

Arbeiter, Achim, Korol, Dieter (Hrsg.), Der Kuppelbau von Centcelles. Neue Forschungen zu einem enigmatischen Denkmal von Weltrang, Tübingen, Wasmuth, 2015.

Baudry, Gérard Henri, Le baptême et ses symboles. Aux sources du salut, Paris, Beauchesne, 2001.

Cipriano, Giuseppina, El-Bagawat. Un cimitero paleocristiano dell'alto Egitto, Todi, Tau editrice, 2008.

Croci, Chiara, Una "questione campana». La prima arte monumentale cristiana tra Napoli, Nola e Capua (secc. IV-VI), Roma, Viella, 2017.

Deichmann, Friedrich Wilhelm, Ravenna: Hauptstadt des spätantiken Abendlandes, Wiesbaden, Steiner, 3 vols, 1969-1989.

Demus, Otto, The mosaics of San Marco in Venice, Chicago, The University of Chicago Press, 1984.

Ferri, Giovanna, I mosaici del battistero di San Giovanni in Fonte a Napoli, Todi, Tau editrice, 2013.

Foletti, Ivan, Gianandrea, Manuela, Zona liminare. Il nartece di Santa Sabina a Roma, la sua porta e l'iniziazione Cristiana, Roma, Viella, 2015.

Gandolfi, Katia, "Les mosaïques du baptistère de Naples: programme iconographique et liturgie", in Il Duomo di Napoli dal paleocristiano all'età angioina, a cura di Serena Romano, Nicolas Bock, Napoli, Electa, 2002, p. 21-34. 
Geymonat, Ludovico, «Parma 1233: pittura e iconografia in un battistero gotico", in L'arte medievale nel contesto 300-1300. Funzioni, iconografia, tecniche, a cura di Paolo Piva, Milano, Jaca Book, 2006, p. 509-515.

Grabar, André, «La décoration des coupoles à Karye Camii et les peintures italiennes du Dugento", Jahrbuch der österreichischen byzantinischen Gesellschaft, 6 (1957), p. 111-123.

Kitzinger, Ernst, "The role of miniature painting in mural decoration", in The place of book illumination in Byzantine art, ed. by Kurt Weitzmann et alii, Princeton, The Art Museum Princeton University, 1975, p. 99-142.

—, "Christian imagery: growth and impact", in Age of spirituality. A symposium, ed. by Kurt Weitzmann, New York, The Metropolitan Museum of Art, 1980, p. 141-163.

Low DEn, John, Early Christian and Byzantine art, London, 1997.

Lubian, Francesco, "Un caso di riscrittura metrica "ultrabreve" dei Vangeli : i distici Miracula Christi», Graecolatina Pragensia, 26 (2016.2), p. 87-109.

MaIer, Jean-Louis, Le baptistère de Naples et ses mosaïques. Étude historique et iconographique, Fribourg, Éditions Universitaires, 1964.

Mango, Cyril, Byzantinische Architektur, Stuttgart, Belser, 1975.

Menna, Maria Raffaella, "I mosaici di Santa Maria Maggiore. Storie dei patriarchi della navata centrale", in L'orizzonte tardoantico e le nuove immagini (312-468), a cura di Maria Andaloro, Milano, Jaca Book, 2006, p. 312-330.

Pelizzari, Gabriele, Il pastore ad Aquileia: la trascrizione musiva della catechesi catecumenale nella cattedrale di Teodoro, San Daniele del Friuli, Edizions Glesie Furlane, 2010.

Rasch, Jürgen J., Arbeiter, Achim, Das Mausoleum der Constantina in Rom, Mainz am Rhein, Verlag Philipp von Zabern, 2007.

Rep. I = Repertorium der christlich-antiken Sarkophage. Rom und Ostia, bearb. von Friedrich W. Deichmann, Wiesbaden, F. Steiner, 1967.

Rep. III = Repertorium der christlich-antiken Sarkophage. Frankreich, Algerien, Tunesien, bearb. von Brigitte Christern-Briesenick, Mainz am Rhein, Ph. von Zabern, 2003.

Spieser, Jean-Michel, Images du Christ. Des catacombes aux lendemains de l'iconoclasme, Paris, Droz, 2015. 
Wilpert, Joseph, Die römischen Mosaiken und Malereien der kirchlichen Bauten vom IV. bis XIII. Jahrhundert, Freiburg i. B., Herder, 1916. Zimmermann, Norbert, Werkstattgruppen römischer Katakombenmalerei, Münster, Aschendorff, 2002.

Crédits iconographiques:

Fig. 1, 4, 5: (C) Domenico Ventura.

Fig. 2: Mango, Cyril, Byzantinische Architektur, Stuttgart, Belser, 1975, p. 224.

Fig. 3: Demus, Otto, The mosaics of San Marco in Venice, Chicago, The University of Chicago Press, 1984, II, tav. 73.

Fig. 6: Repertorium der christlich-antiken Sarkophage. Rom und Ostia, bearb. von Friedrich W. Deichmann, Wiesbaden, F. Steiner, 1967, 42, tav. 13.

Fig. 7: Menna, Maria Raffaella, «I mosaici di Santa Maria Maggiore. Storie dei patriarchi della navata centrale", in L'orizzonte tardoantico e le nuove immagini (312-468), a cura di Maria Andaloro, Milano, Jaca Book, 2006, p. 312-330, fig. 8. 
